\title{
Comparison of Continuous Epidural Blockade and Continuous Femoral Nerve Block on Postoperative Pain in Total Knee Replacement Surgeries: A Prospective Randomised Controlled Study
}

\author{
K.Shanthini ${ }^{1}$, Nishkala Chandra Sekar ${ }^{2}$, Kusuma Mathai ${ }^{3}$ \\ ${ }^{1}$ Specialist Grade 2, Department of Anaesthesiology ESIC medical college \& PGIMSR, K.K.Nagar, Chennai, Tamilnadu, India, ${ }^{2}$ Senior Consultant, \\ Department of Anaesthesiology Southern Railway Headquarters Hospital, Perambur, Chennai, Tamilnadu, India, ${ }^{3}$ Additional Chief Health Director and \\ Head of the Department, Department of Anaesthesiology, Southern Railway hospital, Perambur, Chennai, Tamilnadu, India.
}

\section{Abstract}

Background: The use of epidural analgesia in the management of postoperative pain following orthopedic surgeries has evolved as a critical component of a multimodal approach to achieve the goal of pain relief, early mobilization, and improved compliance with physiotherapy resulting in overall improved outcomes. Aim: The aim of this study is to compare continuous femoral nerve block with continuous epidural block technique for postoperative analgesia in patients undergoing elective total knee replacement surgery. Subjects and Methods: The patients belonging to the ASA I to III scheduled for various knee surgeries under spinal anesthesia were enrolled in this study. They were randomly divided into two equal groups of thirty-three patients each. The Group F patients received continuous femoral nerve blockade and in the Group E patients continuous epidural blocked preoperatively. Results: The analgesic efficacy of both continuous femoral nerve block and continuous epidural nerve block was equal as measured by the visual analogue scores. The incidence of Hypotension was more in the Epidural group. The adverse effects due to the Continuous Femoral Nerve block were lower in comparison with the Continuous Epidural block technique. Conclusion: Continuous femoral nerve blockade provides postoperative analgesia equivalent to that obtained with a continuous epidural blocked but with lesser side effects.

Keywords: Anesthetic technique, continuous femoral nerve blockade, epidural block, postoperative analgesia.

Corresponding Author: Dr. K. Shanthini, Specialist Grade 2, ESIC medical college \& PGIMSR, K. K. Nagar, Chennai, Tamilnadu, India. Email: dr.shanthu@googlemail.com

Received: April 2020

Accepted: April 2020

\section{Introduction}

Postoperative pain management after Total knee replacement remains a challenge due to extensive nerve innervation of the knee joint,the prolonged severe and dynamic component of pain during knee mobilization. Improved analgesia in the immediate postoperative period may decrease long term pain, reduce joint stiffness and improve functional status. ${ }^{[1]}$ Randomized controlled studies suggest that regional techniques provide superior pain relief and faster postoperative knee rehabilitation than systemic analgesia. Blocking the afferent neural stimulus by various neural block techniques with local anaesthetics is very effective in reducing the classical catabolic response to operation. ${ }^{[2]}$ Regional anaesthesia techniques reduce the neuroendocrine stress responses, central sensitization of the nervous system and muscle spasms which occur in response to painful stimuli. Lumbar epidural analgesia has been the technique of choice for postoperative pain management. The advantages of being excellent analgesia both during rest and activity and minimizes systemic narcotic usage and the associated side effects of nausea, vomiting, respiratory depression, pruritus, decreased intestinal motility and urinary retention and can be extended for up to 2 days. ${ }^{[3]}$

Peripheral nerve blocks are becoming the standard of care, because of the ease of institution and enhanced recovery due to the safer side effect profile. Further the concomitant need for anticoagulation in the perioperative period has reports of epidural haematoma, and serious neurological complications. $^{[4]}$

The older patient population and the safety of epidurals along with anticoagulation for a pre-existing medical condition or as prophylaxis for deep vein thrombosis have favoured the development of continuous femoral nerve block. ${ }^{[5]}$

\begin{abstract}
$\underline{\text { AIM }}$
The aim of this study is to compare continuous femoral nerve block with continuous epidural block technique for postoperative analgesia in patients undergoing elective total knee replacement surgery.
\end{abstract}

\section{Subjects and Methods}

Adult Orthopaedic patients coming for unilateral primary 
Total knee replacement surgeries to Southern Railway Hospital in the age group 18 to 80 years were included.

The sample size was based on the results of the previous similar study comparing postoperative analgesia by two different techniques. ${ }^{[6]}$ The mean visual analogue scores and standard deviation were taken from the previous study, and applying a $95 \%$ confidence interval and $80 \%$ power for this study sample size was calculated as $46\{$ i.e $2 \times 23\}$.

To allow for loss of incomplete data collection 66 patients of ASA 1 to 3 undergoing elective unilateral Total knee Replacement were included in the prospective, randomized, controlled study. The study population was divided into two groups of 33 each by a closed envelope randomization schedule.

Group F: Patients who received femoral perineural analgesia for postoperative analgesia $(n=33)$

Group E: Patients who received epidural catheter for postoperative analgesia. $(n=33)$

\section{Inclusion Criteria}

Sixty-six ASA I to III patients coming for elective primary unilateral total knee replacement coming to Southern Railway Headquarters Hospital, of the age group 18 to 80 years.

\section{Exclusion Criteria}

Patient who were unable to give consent due to language problems, Dementia or other conditions that affected the ability to understand pain scales, Renal insufficiency (Creatinine $>1.5 \mathrm{mg} / \mathrm{dl}$ ), Those with ankylosing spondylitis or prior spinal surgeries, moderate to severe COPD, ASA 4, BMI > 35 who were suffering from coagulopathies and bleeding disorders and chronic opioid use were excluded from the study.

Contra-indication to either femoral nerve block (infection overlying the injection site or previous femoropopliteal bypass surgery), or neuraxial block and Peripheral Neuropathies.

The approval from the institutional Ethical Committee was obtained. A thorough preoperative assessment of the patients posted for an elective unilateral total knee replacement was carried out and recorded as given in the proforma. History of the presenting complaint with past history, personal history, prior hospitalization, concurrent medications, History of previous surgery with an emphasis on anaesthesia related complications and allergy to any substance was meticulously done. General and physical examination was done with an emphasis on the contour of the back and area over the inguinal region. Routine investigations were ensured within acceptable limits and any other investigation which was deemed necessary was done and checked. The selection of patients was done based on the inclusion criteria. The selected patients were explained in detail about the procedure and side effects of the study any queries were addressed and willingness to participate in the study were ensured by written informed consent.

Preoperative fasting was ensured for $8 \mathrm{hrs}$ overnight and premedication with Tab Diazepam 5mg and Tab Ranitidine $150 \mathrm{mg}$ was given in the night prior and morning of surgery. An 18G intravenous line was secured and preloading was done with Ringer lactate $10 \mathrm{ml} / \mathrm{kg}$ starting at $7.00 \mathrm{a} . \mathrm{m}$ on the morning of surgery.

In the operation theatre routine anaesthetic and resuscitation equipment, drugs and suction were thoroughly checked. All procedures were done by the same anaesthetist who was the primary anaesthetist but the data collection was done by residents uninvolved with the study, who were familiar with using the visual analogue scores. After explaining the procedure the standard monitoring was applied to the patient. It included continuous oxygen saturation measurement using pulse-oximeter, Non-invasive oscillometric blood pressure measurement, and continuous transthoracic electrocardiography. Patients characteristics were recorded which included baseline heart rate, BP and pain scores on attempted movement. Inpatients, who were assigned to Group A Continuous femoral nerve block was performed by using the landmarks of Winnieet al, ${ }^{[7]}$ with an electrically insulated 22 gauge regional needle (stimuplex, Braun) $5 \mathrm{~cm}$ needle connected to the nerve stimulator. In group B (Epidural), the patient was placed in the sitting position, after sterile preparation and draping an epidural is sited atL2 -L3 intervertebral space or one space higher using $18 \mathrm{G}$ Tuohy needle.

After 20 mins, Group F was tested for femoral nerve block by testing for loss of sensation to cold in the anterior thigh. If there was no loss of sensation the catheter was attempted another time and with another failed attempt, the subject was excluded from the study. Both the groups were given, a standard spinal anaesthetic with $12-15 \mathrm{mg}$ of hyperbaric $0.5 \%$ bupivacaine with $25 \mathrm{mcg}$ Fentanyl in sitting position. The time was noted and the patient was draped for surgery. Standard monitoring was continued and surgery was started after a sensory level of T8 was ensured. All surgeries were performed by the same team and the same methodology. The operative side was raised to allow for venous drainage and the tourniquet was inflated.

The duration of surgery, blood loss and vasopressor required were recorded. All surgeries were over by 12.30p.m. All critical events were treated as appropriate. The routine recording was continued. 20 mins before completion of surgery the appropriate catheter were connected to a syringe infusion pump with $0.2 \%$ Bupivacaine and commenced at $6 \mathrm{ml} / \mathrm{hr}$ and continued for $48 \mathrm{hrs}$ postoperatively. The patient was shifted to post-operative observation ward. Patients were given Inj. Paracetamol $1 \mathrm{gm}$ IV which was continued thrice daily and changed to Tab. Paracetamol $1 \mathrm{gm}$ thrice daily and Tab. Ibuprofen $100 \mathrm{mg}$ twice daily was also continued for 5 days post-surgery.

Post-operative pain was evaluated and comparison done by measurement of visual analogue pain scores and observation of patient vitals in the postoperative period for 48 hours. The primary outcome to be measured was post-operative pain and the secondary outcomes were adverse effects as in incidences of hypotension, bradycardia, vomiting, urinary retention and other side effects of the procedure. Patient satisfaction was measured on a 3 point scale. The pain was measured using a visual analogue scale which was converted to a 5 point score, at 4-hour intervals for a period of $48 \mathrm{hrs}$. The incidence of breakthrough pain and cumulative incidence of tramadol usage were also noted in the post-operative record and critical events were treated as appropriate.

Data analysis was done with the help of a computer using 
Epidemiological Information Package (EPI 2012) developed by the Centre for Disease Control, Atlanta. Using this software range, frequencies, percentages, means, standard deviation, ' $t$ ' value, chi-square and ' $p$ ' values were calculated. Descriptive coefficient statistical analysis has been carried in the present study. To find the difference between two means unpaired t-test has been used. To find the association between two attributes chi-square test has been used.

Concert Diagram Showing the Flow of Patients Through the Study

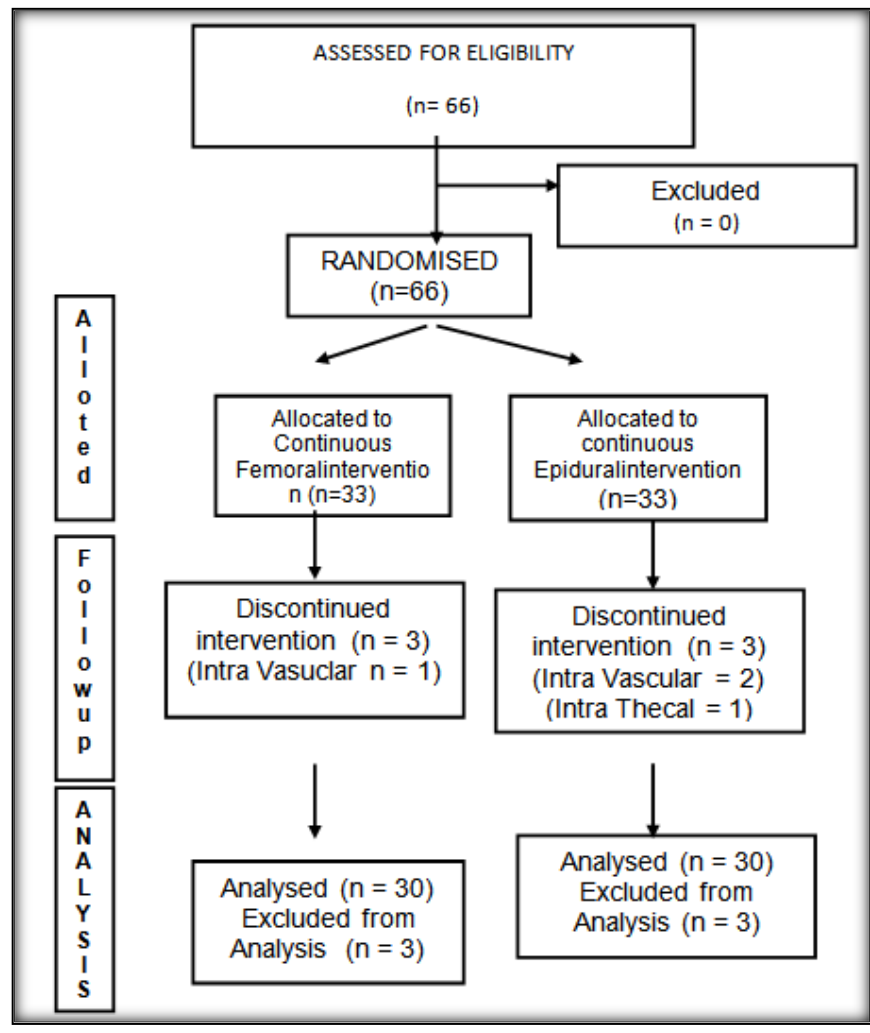

Results

The mean age of persons in Group $\mathrm{F}$ was 60.1 compared to 59.1 in Group E. The distribution of patients in various age groups were comparable in both groups. The majority of the patients were elderly. The distribution of males and females in both groups were comparable. The femoral patients' characteristics as in height, weight and body mass index were comparable. There was no difference in the time taken to site the catheter or in the complications of the procedure. The amount of blood loss as well as the time to completion of surgery were similar. The surgery was done by the same set of surgeons using the same technique and similar implants.

In our study we did not notice any significant difference in the pain level between the two groups as evident in the Visual analogue scores. On the one occasion there was a significant difference in the $2^{\text {nd }}$ postoperative day (i.e. $40 \mathrm{hrs}$ postoperatively).This was also due to the epidural nerve block scoring higher pain levels. The analgesic efficacy of both continuous femoral nerve block and continuous epidural nerve block was equal as measured by the visual analogue scores.

Other variables used to measure analgesic efficacy as in incidences of breakthrough pain and supplemental analgesic required were similar with $\mathrm{p}$-value of 0.5 and 0.694 respectively. The incidence of breakthrough pain was $6.7 \%$ in continuous femoral nerve block whereas it was $10 \%$ in continuous epidural nerve block. They were comparably signifying that there was no advantage of one over another.

The incidence of cumulative tramadol required was also comparable. The incidence was $6.7 \%$ in both groups.

There was a significant difference in the pulse rate and blood pressure between the groups. The incidence of bradycardia in the epidural group was $36.7 \%$ and there were no instances of bradycardia in the femoral group. At the start of surgery the pulse rate was comparable. Throughout the $48 \mathrm{hr}$ postoperative period the pulse rate was lower and the difference was highly significant $p<0.0001$. The presence of the Bezold Jarrisch reflex is the postulated mechanism for bradycardia after central neuraxial block.

The systolic blood pressure at the start of surgery was comparable pre-operatively. (at the start of surgery $\mathrm{p}=0.052$ ). Systolic blood pressures of patients in the epidural group were significantly lower throughout the $48 \mathrm{hr}$ period.. The diastolic blood pressure was comparable in both the groups in many of the instances. On the day of the surgery in the immediate postoperative period, (i.e. for the 1st 24 hours post-surgery). But it was statistically significant on only 2 instances at $8 \mathrm{hrs}$ and $16 \mathrm{hrs}$ post-surgery. The diastolic blood pressure in the epidural group was lower in comparison with the femoral group. The mean arterial pressure was significantly lower on many instances in the 1st $24 \mathrm{hr}$ post-surgery. The incidence of hypotension was $36.7 \%$ in those with epidural, while none developed this complication in with femoral nerve block. This was statistically significant with $\mathrm{p}<0.0001$.

The incidence of vomiting and urinary retention was $16.7 \%$ each in those receiving the epidural.. The increased incidence of urinary retention could be due to the use of $0.2 \%$ bupivacaine in the epidural group, unlike other studies that had used lower concentrations of Bupivacaine. There were no instances of vomiting and urinary retention in those receiving femoral nerve block. There was no other adverse effect as inpatient falls or symptoms of neurological toxicity in both groups.

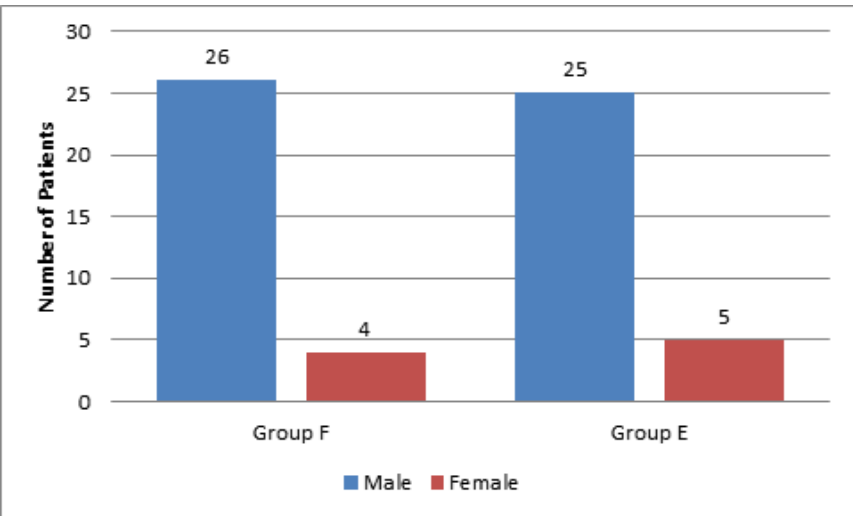

Figure 1: Distribution of Gender

Patient satisfaction was recorded on a 3 point scale. And the 
number of patients who were very satisfied was $63.3 \%$ in those receiving femoral nerve block, which was $10 \%$ in the epidural group. The incidence of persons who were unsatisfied was $3.3 \%$ in the femoral group, which was $40 \%$ in the epidural group. The majority of patients who received the femoral catheter had a satisfying experience. (Very satisfied $=63.3 \%$ and satisfied $=33.3 \%$ ).p $<0.0001$ which denotes high significance.

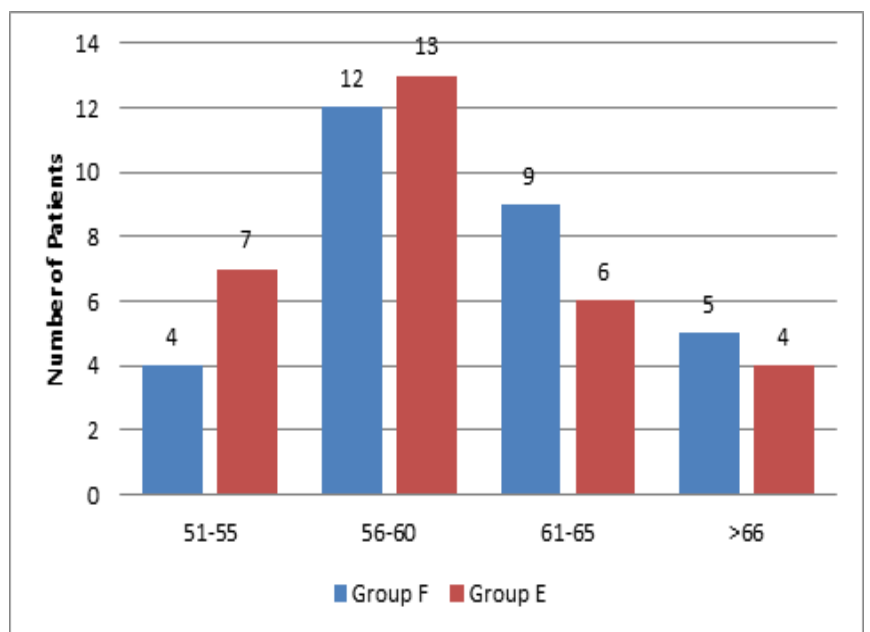

Figure 2: Distribution of Age group

\begin{tabular}{|l|l|l|}
\hline Variables & Group F & Group E \\
\hline BMI & 26.8 & 26.6 \\
\hline Weight & 68.1 & 66.9 \\
\hline Height & 159.3 & 158.6 \\
\hline
\end{tabular}

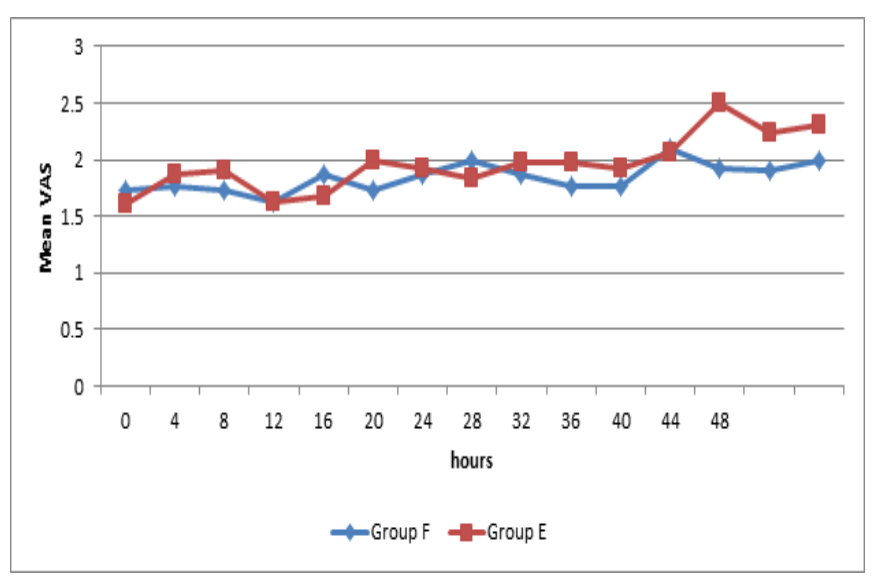

\section{Figure 3: Distribution of patient characteristics}

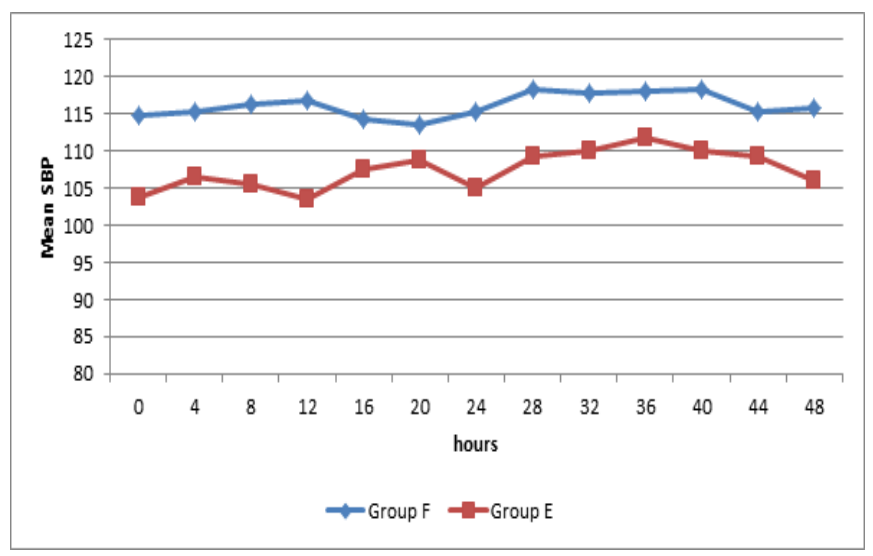

Figure 4: Distributionof Postoperative Visual Analogue Score

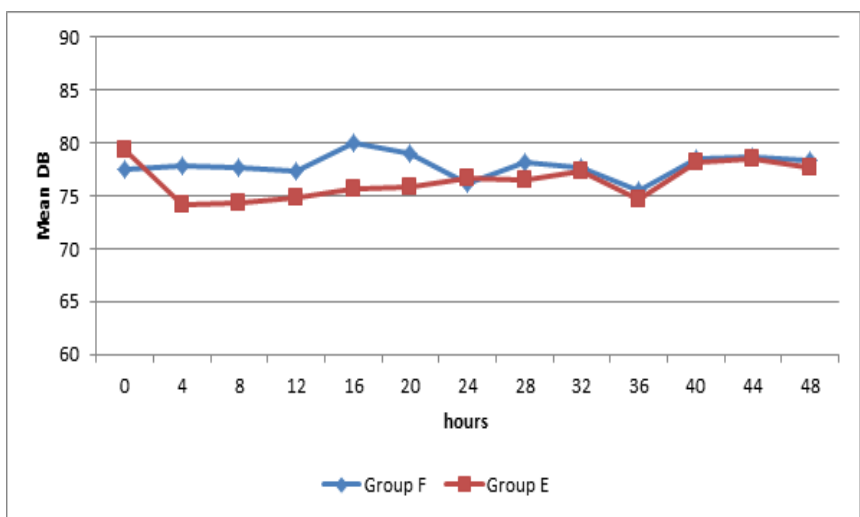

Figure 5: Distribution of Postoperative Mean Systolic Blood Pressure

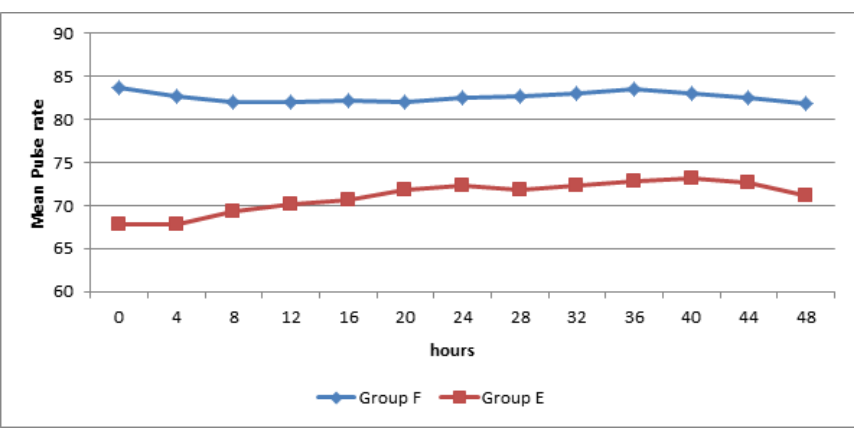

Figure 6: Distribution of Postoperative Mean Diastolic Blood Pressure

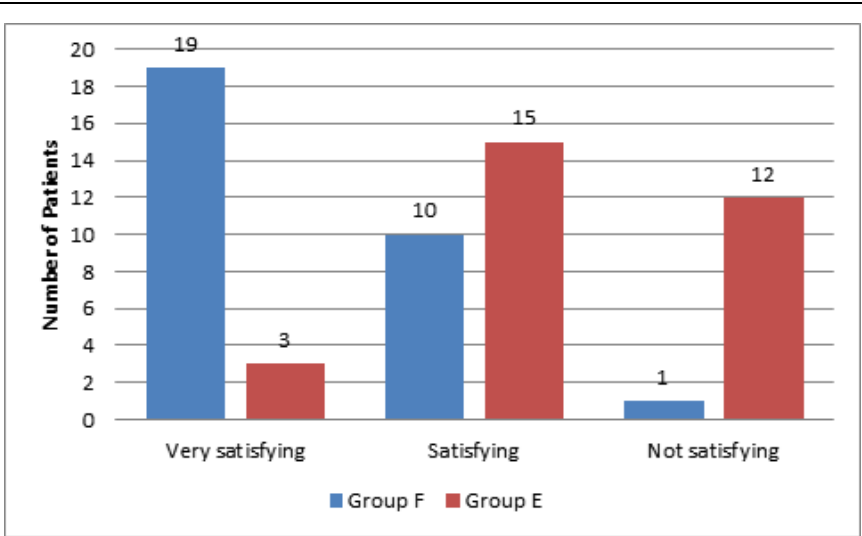

Figure 7: Distribution of Postoperative Mean Pulse rate.

Table 1: Complications

\begin{tabular}{|l|l|l|l|l|l|}
\hline \multirow{2}{*}{$\begin{array}{l}\text { Other } \\
\text { complications }\end{array}$} & \multicolumn{2}{|l|}{ Complications in } & \multirow{2}{*}{ 'p' } \\
\cline { 2 - 5 } & Group A & \multicolumn{2}{|l|}{ Group B } & \\
\cline { 2 - 5 } & No & $\mathbf{\%}$ & No & $\mathbf{\%}$ & \\
\hline Hypotension & - & - & 11 & $36.6 \%$ & $<0.0001$ \\
\hline Vomiting & - & - & 5 & $16.6 \%$ & 0.020 \\
\hline Brady cardio & - & - & 11 & $36.6 \%$ & $<0.0001$ \\
\hline $\begin{array}{l}\text { Others (urine } \\
\text { retention) }\end{array}$ & - & - & 5 & $16.6 \%$ & 0.020 \\
\hline $\begin{array}{l}\text { Any one } \\
\text { complication }\end{array}$ & - & - & 11 & $36.6 \%$ & $<0.0001$ \\
\hline
\end{tabular}

Table 2: Breakthrough pain

\begin{tabular}{|l|l|l|l|}
\hline Breakthrough pain & Present & Absent & P-value \\
\hline Group A & 2 & 28 & 0.64 \\
\hline Group B & 3 & 27 & \\
\hline
\end{tabular}

\section{Discussion}

The aim of postoperative pain treatment is to provide 
subjective comfort in addition to inhibiting trauma-induced nociceptive impulses in order to blunt autonomic and somatic reflex responses to pain and subsequently to enhance restoration of function by allowing the patient to breathe, cough and move more easily. Unrelieved pain after surgery is unhealthy. Postoperative pain limits the ability of the patient for early active and even passive participation in physical therapy delaying recovery, ambulation and hospital discharge. ${ }^{[8]}$

A recent meta-analysis by Paul JE et al. found that singleshot and continuous FNB was superior (lower opioid consumption) to PCA alone. ${ }^{[9]}$ In particular, continuous FNB with intravenous PCA was clearly superior to PCA alone with regard to reduced morphine consumption at 24 and 48 hours, pain scores, and nausea.

Our study shows that analgesic efficacy measured by VAS Scores were comparable and there was no significant difference. However the mean VAS of the Epidural group was lower than the Femoral group up to $24 \mathrm{hrs}$ though not significant and by the end of 24 hours the Femoral group had better control of pain. Barrington et al on studying hundred and twelve patients comparing Continuous Femoral Nerve Block And Epidural Analgesia concluded that there is no difference in pain score between the groups.

The study by Shanthana et al. ${ }^{[10]}$ also shows equivalent analgesia with both CFNB and CEA, but the CFNB group had significantly higher pain scores in the initial 6 hours. In our study the VAS were comparable which could be attributed to the spinal fentanyl which had a prolonged blocking effect. The study by Capdevilla et al ${ }^{[11]}$ was almost similar to our study. The resting VAS of the continuous Epidural group was significantly less than the continuous Femoral Group in the immediate postoperative period. With the progression of time the Femoral Nerve group did better. The significant difference in VAS can be attributed to the use of $1 \%$ lignocaine with 0.03 milligram $/ \mathrm{ml}$ of morphine with $2 \mathrm{mcg} / \mathrm{ml}$ Clonidine in the study by Capdevilla et al. ${ }^{[11]}$ The use of $1 \%$ lignocaine provides better motor blockade than bupivacaine $0.25 \%$ or $0.125 \%$, more effectively avoiding quadriceps muscle spasm, which is cited as the cause and consequence of postoperative pain.

Our choice of $0.2 \%$ Bupivacaine was based on the study by Ganapathy et al. ${ }^{[12]}$ His study concluded that $0.125 \%$ Bupivacaine was ineffective and but that $0.25 \%$ bupivacaine reduced postoperative morphine requirement and improved range of motion.

The Knee is innervated by the lumbosacral plexus. The femoral and obturator nerve through its posterior division innervates the anterior aspect of the knee and the sciatic nerve innervates the posterior aspect. Though blocking of both Femoral and Sciatic nerves may be required to consistently provide postoperative analgesia, postoperative compartmental syndrome causing peroneal nerve injury remains a concern. The study by Allen et al. ${ }^{[13]}$ compared the analgesic efficacy of femoral, Sciatic-femoral and sham nerve blocks after total knee replacement concludes that the addition of a Sciatic nerve block does not provide additional benefit.

Additionally the obturator nerve block has a variable sensory distribution with sometimes no contribution. Thus it can be hypothesized that as the major sensory innervation is provided by the femoral nerve, blocking of this nerve is more effective in providing preventive analgesia and thus avoiding spasm. This needs further in-depth analysis.

Subjects in Group-E had a significantly lower pulse rate in comparison with those having a Femoral Nerve Block. ${ }^{[11]}$ patients in Group E, had significant Hypotension, requiring treatment with ephedrine $3 \mathrm{mg}$. The mean arterial pressure is comparable in both groups. There is a significant difference in the systolic blood pressure between both groups throughout the 72 hour period with group-E recording lower systolic blood pressures. This could have been due to the pronounced loss of sympathetic tone in both the limbs in the epidural group compounded by the blood loss in the postoperative period. However there is no significant difference in the diastolic blood pressure except on one occasion on the 1st postoperative day.

The incidence of Bradycardia is more pronounced in group $\mathrm{E}$, whereas none of the patients in group $\mathrm{F}$ had bradycardia. There were 11 incidences of Bradycardia in Group E who had epidural analgesia for postoperative pain relief.

Bradycardia and asystole can occur unexpectedly during neuraxial anesthesia. Moderate or severe bradycardia may occur at any time during neuraxial anesthesia, regardless of the duration of anesthesia. A low baseline heart rate increases the risk of bradycardia. The reason for the increased incidence of Hypotension in Epidural being the Bilateral blockade of the lower limbs which is being compounded by the huge blood loss involved in total knee replacement surgery.

The meta-analysis by Fowler which compiles the studies of Singelyn et al, Capdevilla et al. and Barrington et al. state that the incidence of hypotension is more in subjects having Epidural than in the group with peripheral nerve blockade. Odds ratio $0.19(0.08,0.45){ }^{[6,11,14]}$

There was a significant difference in the incidence of vomiting between the two groups as evident from the Pvalue. Five patients out of group B who developed hypotension had vomiting (incidence 16\%). The vomiting occurred during the episode of hypotension. None of the patients in group A had vomiting.

The most common reason for nausea being hypotension during anesthesia. Other causes are drug-induced particularly opioids. Hypotension is a common occurrence during neuraxial anesthesia and could be a result of direct neurogenic stimulation of the vomiting centre. ${ }^{[14]}$ Other postulated mechanisms include low blood pressure which may lead to brain stem ischemia, which is thought to activate the circulatory, respiratory, and vomiting centers grouped together in the medulla. Hypotension also leads to gut ischemia and the release of emetogenic substances (e.g., serotonin) from the intestines. Neuraxial anesthesia also changes the function of the gastrointestinal tract. Sympathetic blockade by local anesthetics creates unopposed vagal action, resulting in gastrointestinal hyperactivity.

In our study, 5 patients belonging to Group E had urinary retention, requiring catheterization. None of the patients in Group F developed urinary retention. Patient satisfaction was more with group $\mathrm{F}$ compared with group $\mathrm{E}$, and it was found to be clinically significant from the $\mathrm{P}$-value. The most common reason found for the decreased acceptance in group $\mathrm{E}$ was that most patients don't like to have a catheter at the 
back even though the pain relief was good. The unilateral analgesia giving a sense of well being and independence is the most common reason for patients preferring this technique.

In summary, the added value of Continuous femoral nerve block for postoperative pain control in total knee replacement surgery is providing targeted analgesia, which can be adjusted to individuals with different programmes of rehabilitation. More effective for dynamic pain and concentration can be increased and titrated according to individual requirements.

Additionally postoperative joint inflammation and inflammatory markers may be decreased. There is likely benefit in prevention of deep vein thrombosis, due to the increased blood flow in the area of the unilateral sympathectomy, and the effects of regional anaesthesia on coagulation and fibrinolysis cascades secondary to the altered neuroendocrine response to surgery. In addition, the inhibitory effects of local anaesthetics on platelet adhesion, aggregation, and release may be clinically useful, as demonstrated by Cooke et al, ${ }^{[16]}$ Complications from femoral nerve catheters are few. A prospective observational study followed 208 patients for the incidence of infectious, vascular and neurological complications after placement of a continuous femoral nerve catheter via either the femoral nerve sheath approach or the fascia iliac approach. Although there was a frequent incidence of catheter colonization (57\%) only 3 patients presented with fever and bacteraemia and they did not require antibiotic therapy.

The benefits of continuous femoral nerve catheters are haemodynamic stability because of unilateral sympathectomy and technical feasibility in patients with fused spines and obese individuals and usage in the ambulatory setting. They do not require positioning and can be done in the supine position. Further, severe neurological complications as spinal hematoma, cauda equina syndrome have been reported after the central neural blockade. Peripheral nerve blocks can be safely performed in both anticoagulated and septic patients.

\section{Conclusion}

The Analgesic efficacy of Continuous Femoral Nerve Block when compared with Continuous Epidural analgesia for postoperative pain in Total knee replacement was equal. The adverse effects due to the Continuous Femoral Nerve block were lower in comparison with the Continuous Epidural block technique. Additionally patient satisfaction was higher in Continuous Femoral Group on comparison with Continuous Epidural group. So as continuous Femoral nerve has a better side effect profile and a better sense of subjective wellbeing, it is recommended as the analgesic technique for postoperative pain in total knee replacement surgeries.

\section{Limitations}

This study was non-blinded as it would not be ethical to conduct a sham epidural and sham nerve block in the subjects.

\section{References}

1. Allen JG, Denny NM, Oakman N. Postoperative analgesia following total knee arthroplasty: a study comparing spinal anesthesia and combined sciatic femoral 3- in-1 block. Reg Anesth Pain Med. 1998 Apr 30;23(2):142-6

2. Davies AF, Segar EP, Murdoch J, Wright DE, Wilson IH. Epidural infusion or combined femoral and sciatic nerve blocks as perioperative analgesia for knee arthroplasty. Br J Anaesth. 2004 Sep 1;93(3):368-74.

3. Kehlet $H$. Multimodal approach to control postoperative pathophysiology and rehabilitation. Br J Anaesth. 1997 May;78(5):60617.

4. Bondar A, Morau D, Kollipara S, Iohom G. Peripheral nerve blockade. Anesthesiol Res Pract. 2011;2011:973239. doi:10.1155/2011/973239

5. Liu J, Yuan W, Wang X, et al. Peripheral nerve blocks versus general anesthesia for total knee replacement in elderly patients on the postoperative quality of recovery. ClinInterv Aging. 2014;9:341-350.

6. Singelyn FJ, Deyaert M, Joris J, Pendeville E, Gouverneur JM. Effects of intravenous patient-controlled analgesia with morphine, continuous epidural analgesia, and continuous three-in-one block on postoperative pain and knee rehabilitation after unilateral total knee arthroplastie. Anesth Analg $1998 ; 87: 88-92$.

7. Winnie AP, Ramamurthy S, Durrani Z. The inguinal paravascular technic of lumbar plexus anesthesia: the" 3-in-1 block". Anesth Analg. 1973 Nov 1;52(6):989-96.

8. Duarte, Domingues LT, Ângelo SR. Effects of epidural analgesia and continuous lumbar plexus block on functional rehabilitation after total hip arthroplasty. Revista Brasileira de Anestesiologia 2009;59(5):531544.

9. Paul JE, Arya A, Hurlburt L, Cheng J, Thabane L, Tidy A, Murthy Y. Femoral Nerve Block Improves Analgesia Outcomes after Total Knee Arthroplasty. A Meta-analysis of Randomized Controlled Trials. J Am Soc Anesthesiol. 2010 Nov 1;113(5):1144-62.

10. Shanthanna H, Huilgol M, Manivackam VK, Maniar A. Comparative study of ultrasound-guided continuous femoral nerve blockade with continuous epidural analgesia for pain relief following total knee replacement. Indian J Anaesth. 2012 May;56(3):270.

11. Capdevila X, Coimbra C, Choquet O. Approaches to the lumbar plexus: success, risks, and outcome. Reg Anesth Pain Med. 2005 Mar 1;30(2):150-62.

12. Ganapathy S, Wasserman RA, Watson JT, Bennett J, Armstrong KP, Stockall CA, Chess DG, MacDonald C. Modified Continuous Femoral Three-in-One Block for Postoperative Pain After Total Knee Arthroplasty. Anesth Analg. 1999 Nov 1;89(5):1197-202

13. Allen HW, Liu SS, Ware PD, Nairn CS, Owens BD. Peripheral nerve blocks improve analgesia after total knee replacement surgery. Anesth Analg. 1998 Jul 1;87(1):93-7.

14. Borgeat A, Ekatodramis G, Schenker CA. Postoperative Nausea and Vomiting in Regional Anesthesia: A Review. J Am Society Anesthesiol. 2003 Feb 1;98(2):530-47.

15. Barrington MJ, Olive D, Low K, Scott DA, Brittain J, Choong P. Continuous femoral nerve blockade or epidural analgesia after total knee replacement: a prospective randomized controlled trial. Anesth Analg. 2005 Dec 1;101(6):1824-9.

16. Cooke ED, Lloyd MJ, Bowcock S, Pilcher MF. Intravenous lignocaine in prevention of deep venous thrombosis after elective hip surgery. The Lancet. 1977 Oct 15;310(8042):797-9. 
Copyright: (C) the author(s), 2020. It is an open-access article distributed under the terms of the Creative Commons Attribution License (CC BY 4.0), which permits authors to retain ownership of the copyright for their content, and allow anyone to download, reuse, reprint, modify, distribute and/or copy the content as long as the original authors and source are cited.

How to cite this article: Shanthini K, Sekar NC, Mathai K. Comparison of Continuous Epidural Blockade and Continuous Femoral Nerve Block on Postoperative Pain in Total Knee Replacement Surgeries: A Prospective Randomised Controlled Study. Acad. Anesthesiol. Int. 2020;5(1):67-73.

DOI: dx.doi.org/10.21276/aan.2020.5.1.14

Source of Support: Nil, Conflict of Interest: None declared. 